\title{
The Zoonoses Data Collection in Italy: An Expert System for Data Quality Management and Improvement
}

\author{
lannetti $\mathbf{S}^{1 *}$, Cioci $\mathbf{D}^{2}$, Falcone $\mathbf{M G}^{3}$ and Colangeli $\mathbf{P}^{2}$ \\ ${ }^{1}$ Veterinary Medicine, Istituto Zooprofilattico Sperimentale of Abruzzo and Molise G. Caporale, Campo Boario Teramo, Italy \\ ${ }^{2}$ Computer Science, Istituto Zooprofilattico Sperimentale of Abruzzo and Molise G. Caporale, Campo Boario Teramo, Italy \\ ${ }^{3}$ Veterinary Medicine, Ministry of Health, Office III DGSAF, Rome, Italy
}

\begin{abstract}
The need to improve the quality of data for a better analysis and understanding of zoonoses' trend at country level has been increased year by year both by the EFSA and by reporting countries. In the framework of an EFSA's Grant project, aimed to complement the zoonoses historical database, an expert system based on logical rules of truth tables was put in place within the Italian information system for zoonoses data collection (SINZoo). During data entry, the truth tables check that, for each zoonoses, the combination of the area of interest, each possible sampling context, stage and sampling unit has been entered correctly, thus avoiding inconsistent data. Each combination available in the truth tables indicates the context, the stage, the sampling unit allowed for each zoonosis in a specific area and for a category of species. The goal of the project was achieved for most of the information to be retrieved: the $89 \%$ and the $83 \%$ of sampling contexts and stages respectively and the $100 \%$ of the other information were retrieved. To date, the truth tables developed for specific zoonoses have become integral part of SINZoo, allowing to avoid mistakes during data reporting. Data quality is the pillar for any analysis and to perform risk analysis: the logical rules of truth tables can be implemented in other information systems involved in data collection in the field of animal health and food safety, increasing both the consistence and the coherence of the data reported.
\end{abstract}

Keywords: Business rules; Data collection; Data quality; Data dictionary; Expert system; Information system; Zoonoses

\section{Introduction}

The European Union (EU) system for monitoring and collecting information on zoonoses is based on Directive (EC) n. 2003/99, which obliges the EU Member States to collect data on the occurrence of zoonoses, zoonotic agents, antimicrobial resistance, animal populations and food-borne outbreaks [1]. Moreover, under Regulation (EC) $\mathrm{n}$. $178 / 2002$, the European Food Safety Authority (EFSA) is assigned the tasks of examining these data and publishing annual European Union Summary Reports (EUSR) in cooperation with the European Centre for Disease Prevention and Control (ECDC), which provides and analyses the data on zoonotic infections in humans [2]. In order to collect data by the different Member States, which already have different systems to collect zoonoses data, EFSA developed a Zoonoses Data Model and a common dictionary (contained in pick lists) to be used by each reporting country, based on the Standard Sample Description, for all the items to be reported, with the aim of collecting data in a unique and uniform way and with the same semantic [3].

In Italy, the Italian Ministry of Health $(\mathrm{MoH})$ is the national authority in charge of collecting data on zoonoses and zoonotic agents covered by Directive (EC) n. 2003/99 [4]. Since 2008, zoonoses data collection takes place at national level through the "national information system for zoonoses data collection", named SINZoo, developed in compliance with the Directive (EC) n. 2003/99 by the Istituto Zooprofilattico Sperimentale dell'Abruzzo e del Molise "G. Caporale" (IZSAM) on request of the MoH. Zoonoses data are registered into SINZoo by the regional veterinary services through different alternatives: on line forms, upload of XML files and Web Services [5]. A common dictionary was developed in SINZoo, integrated with the EFSA's one and with other categorizations specifically developed at national level. The database in SINZoo was established considering both the information debt toward the EFSA and the national needs and is made up of the following components:

-Zoonosis
- Tested species for animal prevalence data, species of origin of the food for food prevalence data, species of destination of feed for feed prevalence data

- Sampling Context

- Sampling Stage/Sample Type

- Sampling Unit

Each component includes a list of possible values which found the correspondence (mapping) of the values in the EFSA's catalogue [6].

SINZoo is strictly integrated with other national information systems already existent in animal health and food safety and is available on the website of the National Veterinary Information systems, thus avoiding double data entry and assuring data uniqueness and the coherence between dictionaries and definitions [7-9]. In fact, the national information debt towards the EFSA consists in aggregated data (i.e., not sample based) which come from the different national information systems:

- Data on animal population, available in the National veterinary database for livestock and holdings (BDN),

- Data on zoonoses and zoonotic agents in animal, food and feed,

*Corresponding author: lannetti S, Veterinarian, Doctor in Veterinary Medicine, Research Institute of Abruzzo and Molise G. Caporale, Campo Boario Teramo, Italy, Tel: 00390861332243; E-mail: s.iannetti@izs.it

Received February 17, 2017; Accepted March 15, 2017; Published March 17, 2017

Citation: lannetti S, Cioci D, Falcone MG, Colangeli P (2017) The Zoonosis Data Collection in Italy: An Expert System for Data Quality Management and Improvement. J Vet Sci Technol 8: 431. doi: 10.4172/2157-7579.1000431

Copyright: ( $\odot 2017$ lannetti S, et al. This is an open-access article distributed under the terms of the Creative Commons Attribution License, which permits unrestricted use, distribution, and reproduction in any medium, provided the original author and source are credited. 
that represent the "prevalence data", entered in SINZoo by the authorised users,

- Data on Community co-financed eradication programs on sheep and goat brucellosis and bovine tuberculosis, collected by the Italian information system on Community co-financed eradication programmes (SIR),

- Data on the national Salmonella control program, collected by the Italian National information system for Salmonella control programme,

- Antimicrobial resistance data, which are provided by the national reference centre for antimicrobial resistance,

- Food-borne outbreak data, entered in SINZoo at outbreak level by the authorised users of the Local Health Units.

In order to improve data quality, a national Panel of 15 experts was appointed by the MoH for the evaluation of data collected prior to their submission to the EFSA. Despite this, during the first years, the lack of automatic controls increased data granularity and inconsistencies. Moreover, the need to improve data quality for a better analysis and understanding of the trend of zoonoses at country level increased year by year, both by the EFSA and by reporting countries: this was the reason why the EFSA launched in 2014 a Grant project within the article 36 of EFSA's Founding Regulation, to support the participating countries in updating and complementing their datasets in EFSA's historical databases. In the framework of this Grant project (Implementation and testing of electronic submission in XML, Excel and CSV formats of zoonoses, antimicrobial resistance and food-borne outbreak data and updating the historical dataset) the EFSA provided funds to the IZSAM for updating and revising the national historical dataset on zoonoses and zoonotic agents in animals from 2008 to 2011. In this context, an Expert system based on logical rules and truth tables was put in place in SINZoo for complementing the historical animal prevalence data and improving the overall data quality.

The aim of this paper is to describe the logical rules behind the Expert system and the results obtained.

\section{Materials and Methods}

The EFSA's historical database on animal prevalence data was firstly evaluated by year, then data with incorrect/missing values were identified and categorized. The inconsistencies consisted in missing or unspecified information to retrieve and wrong information to correct, in relation to the sampling context (which was split by EFSA during the reporting years in three fields: context, sampler, sampling strategy), sampling stage, sample type, sampling unit. The missing or unspecified information found were retrieved following a set of logical rules based on the legislation in place regarding the zoonoses covered by the project. After defined the logical rules, the removal of rows with clear errors in the EFSA's historical database was made possible by implementing in SINZoo truth tables customized for each zoonosis. In order to retrieve missing or incorrect data in the EFSA's historical database, it was considered worthwhile to analyse not all records, but only substantial combinations (SCs) of: matrix, sampling context, sampling strategy, sampler, sample type, sampling stage, sampling unit. Each SCs was substituted with a new combination where missing or incorrect field/s were replaced according to the logical rules. The SCs to be corrected were modified through a web interface, integrated with SINZoo, available only to users having a specific role (i.e., epidemiologists involved in the Grant project). The web interface allowed filtering the SCs through a search-form, to display them and to modify each combination manually. Once saved the correction, a $\mathrm{pl} / \mathrm{sql}$ procedure verified if the new combination still contained errors/warning; in this case, the user was able to correct the SC for unlimited times. The data updating was performed in two different ways:

- Massive update: Allowed updating all rows belonging to a SC. Starting from a generic combination, the system allowed modifying (with the same criteria) all rows belonging to it.

- Single row update: Allowed updating a specific row which lies outside the rules foreseen for its SC and must be treated differently. Starting from the generic SC, the system allowed showing all single rows having that attributes' combination and permitted and modify a specific row in a way and all the others in a different way.

After data updating, some row amended could have been acquired the same SC belonging to other rows already existing into the EFSA's historical database. In this situation, data of the "new" and "old" combinations were automatically aggregated and total number of unit tested and total number of units positive was summed.

\section{Examples of logical rules developed for missing, unspecified, wrong sampling context}

In Italy, according to the Legislative Decree 4 April 2006 n. 191, the zoonoses to be monitored by the official competent authority are listed in the Annex I part A. Depending on the epidemiological situation of the region, other zoonoses shall be monitored and they are listed in Annex I part B. Therefore, for zoonoses and zoonotic agents listed in the Annex I part A, regarding animal area, when the sampler reported was "official sampling" and the context was "unspecified", the context was updated to "monitoring"; vice versa, when the context reported was "monitoring/ surveillance" and the sampler was unspecified, the sampler was updated to "official sampling".

National control and co-financed eradication programmes are in place for salmonellosis in Gallus gallus, tuberculosis due to M. bovis in cattle and water buffaloes, brucellosis in cattle, in water buffaloes and in sheep and goats. Thus, all the official sampling activities are carried out for the purpose of control and co-financed eradication programmes. When the context reported was "control and eradication programme" and the sampler was "official sampling", the missing sampling strategy was updated to "census", according to the legislation in place related to the national control and co-financed eradication programmes $[10,11]$.

For official sampling activities in Gallus gallus and sampler "official sampling" the missing sampling context was updated to "control and eradication programme", and the sampling unit to "flock" if missing or reported with other values, since the controls are performed at flock level [12-15].

For trichinosis, according to Commission Regulation n. 2075/2005 and subsequent amendments laying down specific rules on official controls for Trichinella in meat, carcases of domestic swine shall be systematically sampled in slaughterhouses as part of the postmortem examination [16]. Moreover, carcases of horses, wild boar and other farmed species susceptible to Trichinella infestation shall be systematically sampled in slaughterhouses, while carcases of wild animal shall be tested in game-handling establishments as part of the post-mortem examination. Therefore, the logic rule implemented for this zoonosis was: in farmed animals, when the sampler was "official sampling" the sampling strategy should be "census" and the sampling 
stage should be "at slaughterhouse", while in wildlife the sampling stage should be "at game handling establishments".

Data on Toxoplasma, Yersinia, Staphylococcus, Campylobacter and Coxiella in animals are mainly collected during clinical investigations, or surveys, since for the years covered by the project there was not a national monitoring system in place for these zoonoses in Italy. Therefore for such zoonoses the only possible sampling context in the EFSA's historical database could be "survey" or "clinical investigation". Clinical investigations are usually carried out for testing of symptomatic animals suspected to be affected by any zoonotic agent, therefore the sampling strategy was updated to "suspect sampling" when missing. The sampling context was also revised in case of mistakes: the main corrections regarded for instance official sampling activities reported wrongly with sampling context "monitoring" or "surveillance" for which the sampling context was amended and the sampling strategy was corrected to "objective sampling" or to "suspect sampling" in case of surveys or clinical investigations respectively.

\section{Examples of logical rules developed for missing, unspecified, wrong sampling stage}

The sampling stage was updated to "at farm" in case of farmed animals or of zoo animals, (since this stage in SINZoo was intended to be used for all type of premises keeping animals). As regards pets, in Italy most of them are investigated at private level; to date, EFSA does not include this stage in the pick lists, so it was left to "unspecified" in the EFSA's historical database. For wild animals, the only possible sampling stages could be "natural habitat", "conservation facilities" or "game handling establishments", depending on the zoonosis, the tested species and other rules described previously.

\section{Examples of logical rules for generic species}

EFSA implemented the matrix pick list within its catalogue, asking Member States to specify if a given species was farmed or wild, in order to obtain more and more detailed data. Therefore, a logical rule was implemented for wild boars and water buffaloes to decide whether the animals sampled were farmed or not. So, in case of water buffalo, this species was updated to "water buffalo-farmed" and the stage was updated to "at farm" when unspecified or missing, since this species in Italy is only farmed (Table 1). For Echinococcus and Trichinella in water buffaloes and in wild boars-farmed, the only possible sampling stage could be "at slaughterhouse", since according to the legislation, these species shall be systematically sampled at the slaughterhouse [16], while for wildlife-wild boars, the only possible sampling stage could be "natural habitat", where the animals can be hunted (Table 2). The correction of the reported species regarded "pigs-mixed herds-sows", which were corrected to "pigs - breeding animals - unspecified sows" and "pigs-mixed herds-fattening pigs" were corrected to "pigs fattening pigs - unspecified", since there are not mixed herds of farmed pigs in Italy.

\section{Results}

The logical rules described previously were used to create a truth table for each zoonosis to be reported to the EFSA. Each truth table was implemented taking into account the relationship between the zoonosis, the area of interest (animal, food, and feed) and each possible sampling context, stage and sampling unit. From Decoding menu of SINZoo, it is possible to download truth tables for each of the zoonoses to be reported to the EFSA according to the Dir. 2003/99/CE (Figure 2). The entire EFSA's historical database was processed by the truth tables in order to extract the rows containing combinations fields which were not admitted. Each time one or more row was updated, the system verified its correctness by checking the truth table for the zoonosis in question.

The database of SINZoo is logically structured within the entityrelationship (E/R) schemas (Figure 1). The truth table is linked, through foreign keys, to:

- zoonosis,

\begin{tabular}{|c|c|c|c|c|c|}
\hline COD_EFSA & DESCR_EFSA & stage & stage update to: & species updated to: & species considered as: \\
\hline A003581A & Wildlife - wild boars & unspecified or null & natural habitat & & wild animals \\
\hline A020861A & Wild boars - farmed & unspecified or null & at farm & & Farmed/zoo animals \\
\hline A010041A & Wild boars & at farm & & wild boars - farmed & Farmed/zoo animals \\
\hline A010041A & Wild boars & unspecified or null & natural habitat & Wildlife - wild boars & wild animals \\
\hline A003581A & Wildlife - wild boars & at farm & natural habitat & & wild animals \\
\hline A020861A & Wild boars - farmed & natural habitat & at farm & & Farmed/zoo animals \\
\hline $\mathrm{A} 006821 \mathrm{~A}$ & Water buffalos & unspecified or null & at farm & Water buffalos - farmed & Farmed/zoo animals \\
\hline A006821A & Water buffalos & at farm & & Water buffalos - farmed & Farmed/zoo animals \\
\hline A031641A & Water buffalos - farmed & unspecified or null & at farm & & Farmed/zoo animals \\
\hline A031641A & Water buffalos - farmed & natural habitat & at farm & & Farmed \\
\hline
\end{tabular}

Table 1: Logical rules followed to update generic species.

\begin{tabular}{|c|c|c|c|c|c|}
\hline COD_EFSA & DESCR_EFSA & stage & stage update to: & species updated to: & species considered as: \\
\hline $\mathrm{A} 003581 \mathrm{~A}$ & Wildlife - wild boars & unspecified or null & natural habitat & & wild animals \\
\hline $\mathrm{A} 003581 \mathrm{~A}$ & Wildlife - wild boars & at farm & natural habitat & & wild animals \\
\hline A010041A & Wild boars & at farm & at slaughterhouse & wild boars - farmed & Farmed/zoo animals \\
\hline A010041A & Wild boars & unspecified or null & natural habitat & Wildlife - wild boars & wild animals \\
\hline A020861A & Wild boars - farmed & unspecified or null & at slaughterhouse & & Farmed/zoo animals \\
\hline A020861A & Wild boars - farmed & natural habitat & at slaughterhouse & & Farmed/zoo animals \\
\hline A006821A & Water buffalos & unspecified or null & at slaughterhouse & Water buffalos - farmed & Farmed/zoo animals \\
\hline A006821A & Water buffalos & at farm & at slaughterhouse & Water buffalos - farmed & Farmed/zoo animals \\
\hline A031641A & Water buffalos - farmed & unspecified or null & at slaughterhouse & & Farmed/zoo animals \\
\hline A031641A & Water buffalos - farmed & natural habitat & at slaughterhouse & & Farmed/zoo animals \\
\hline
\end{tabular}

Table 2: Logical rules followed to update generic species in case of Echinococcus and Trichinella. 
Citation: lannetti S, Cioci D, Falcone MG, Colangeli P (2017) The Zoonosis Data Collection in Italy: An Expert System for Data Quality Management and Improvement. J Vet Sci Technol 8: 431. doi: 10.4172/2157-7579.1000431

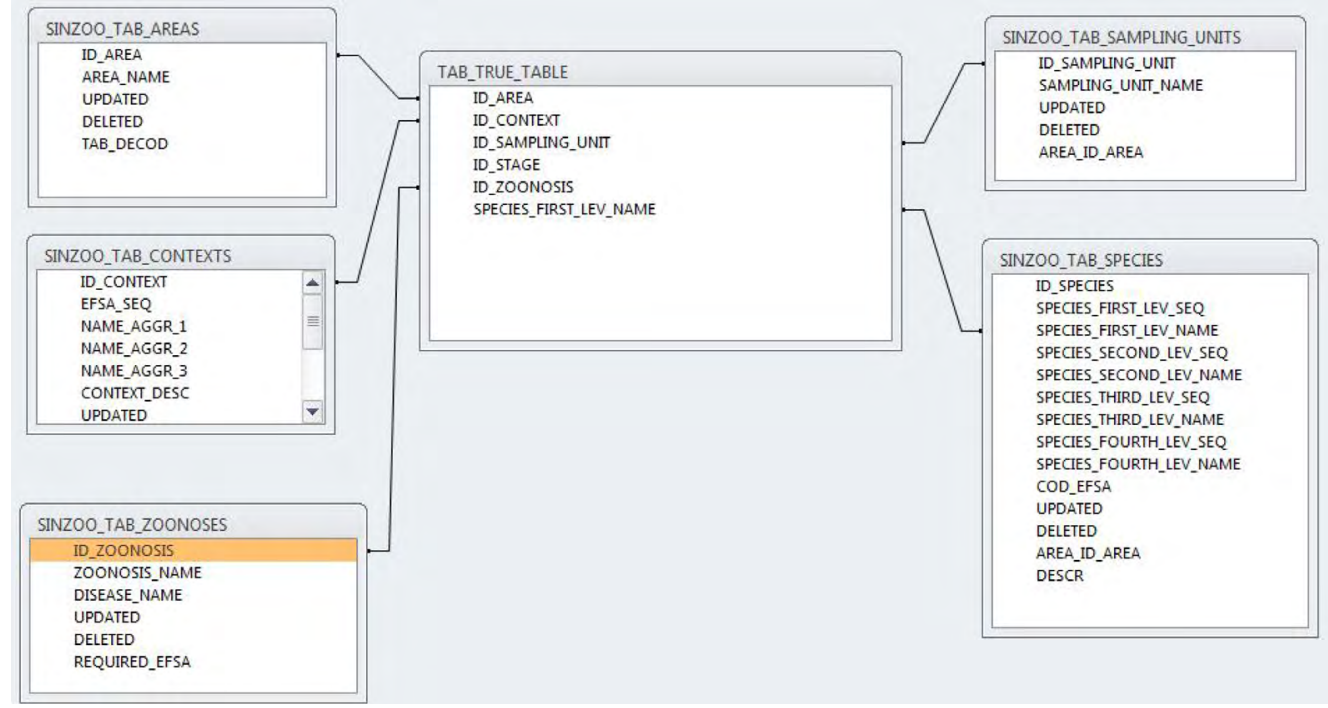

Figure 1: Entity-relationship (E/R) scheme.

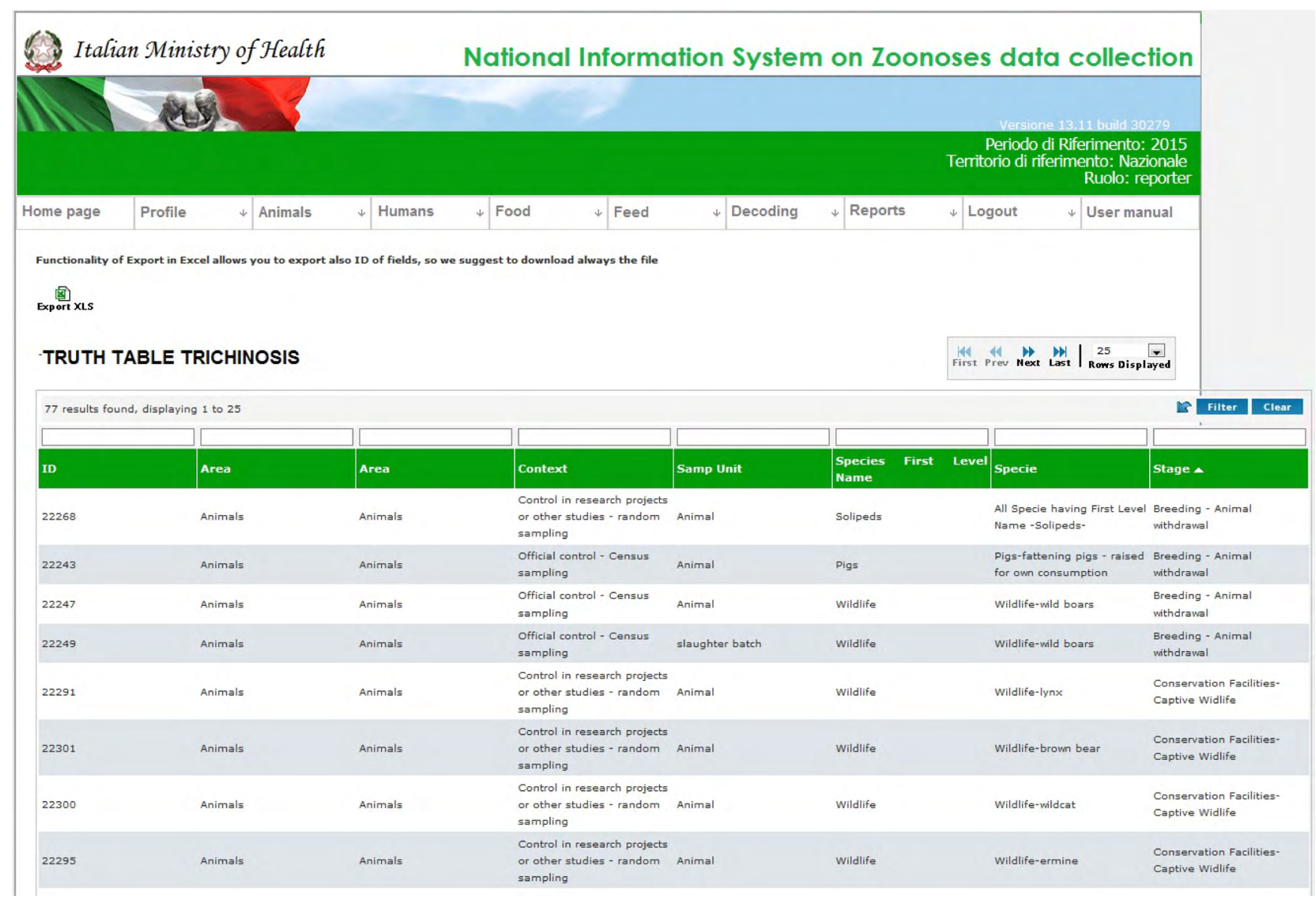

Figure 2: Truth table trichinosis. 
Citation: Iannetti S, Cioci D, Falcone MG, Colangeli P (2017) The Zoonosis Data Collection in Italy: An Expert System for Data Quality Management and Improvement. J Vet Sci Technol 8: 431. doi: 10.4172/2157-7579.1000431

Page 5 of 7

- area,

- context,

- $\quad$ stage,

- $\quad$ sampling unit,

- Species (first level name): this field stands for the animal species if the reporting area is "Animal", the species of origin of the food if the area is "Food" and the species of destination of the feed if the area is "Feed".

Each combination available in the truth table indicates the context, the stage, the sampling unit allowed for the reported zoonosis in a specific area and for a category of species. Thanks to the logical rules and the truth tables, the missing/unspecified information was retrieved and the rows containing wrong combinations of zoonosis/ context/ stage/unit were corrected and updated. Overall, the goal of the Grant project was achieved for most of the information to be retrieved: the $89 \%$ and the $83 \%$ of sampling contexts and stages respectively and the
$100 \%$ of the other information were retrieved (Table 3). The entire process was realised following logical rules both of the truth table and of the national legislation in place for the zoonoses covered by the project, thus ensuring the coherence of all the data retrieved. Also, the whole work was made easier by the implementation of the web interface shown in Figure 3: an alert "message" notified if the record selected had different or equal values for the fields composing the "context", i.e., sampler/ sampling context/ sampling strategy and what type of values. The user might change the selected values and by clicking on "Confirm select", saved the modification. Contextually, the system processed new combination and re-generates the comment field with success/error/ warning (Figure 3).

\section{Examples of truth table developed for some specific zoonosis and zoonotic agent}

Toxoplasma, Yersinia, Staphylococcus, Campylobacter, Coxiella: National monitoring programme for Toxoplasma, Yersinia, Staphylococcus, Campylobacter and Coxiella in animals are not in place

\begin{tabular}{|c|c|c|c|c|c|c|}
\hline Reporting Year & Sampling Context & Sampler & Sampling Strategy & Sampling Stage & Sample Type & Sampling Unit \\
\hline 2008 & $100 \%$ & $100 \%$ & $100 \%$ & $80 \%$ & - \\
\hline 2009 & $99 \%$ & $100 \%$ & $100 \%$ & $86 \%$ & $100 \%$ \\
\hline 2010 & $100 \%$ & $100 \%$ & $100 \%$ & $80 \%$ & $100 \%$ \\
\hline 2011 & $87 \%$ & - & - & $87 \%$ & $100 \%$ \\
\hline Total & $\mathbf{8 9} \%$ & $\mathbf{1 0 0} \%$ & $\mathbf{1 0 0} \%$ & $\mathbf{8 3} \%$ & $\mathbf{1 0 0 \%}$ \\
\hline
\end{tabular}

Table 3: Percentage of retrieved information for each SCs.

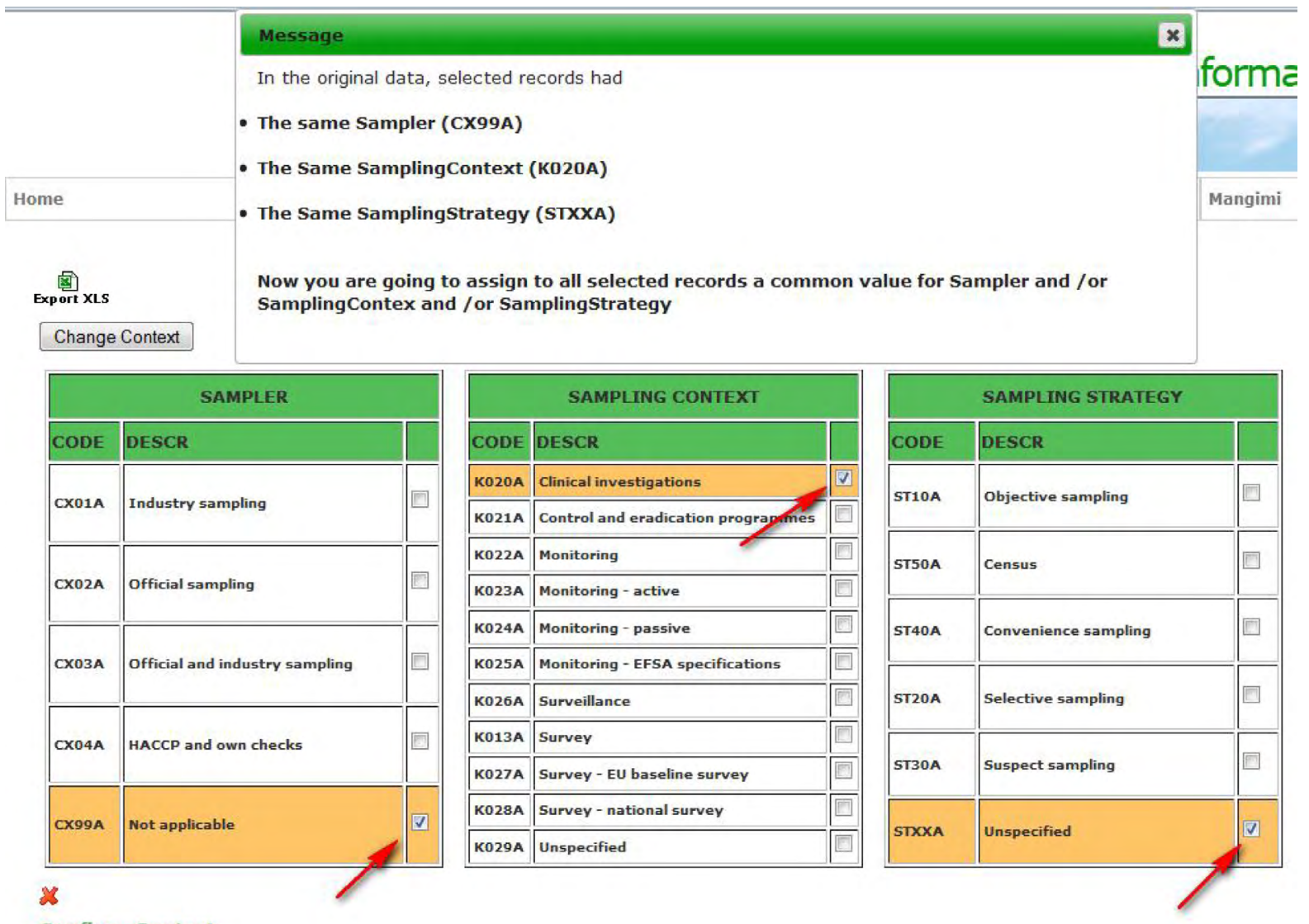

Figure 3: Web inferace. 
for susceptible species, so most of the sampling activities are carried out during surveys or clinical investigations in animals suspected to be diseased, therefore for such zoonoses the truth tables admit only two sampling context (and only the susceptible species), "survey" or "clinical investigation". Moreover, in case of Campylobacter in poultry and birds, Campylobacteriosis does not cause clinical signs, therefore the truth table does not admit "clinical investigations" in these species. The sampling strategy admitted for clinical investigation is "suspect sampling", since the animals are controlled in case of suspectd disease. Also sample type admitted are coherent with the zoonosis: i.e., for Toxoplasma, typically blood (for intermediate hosts) is tested by serology, other samples could include abortion material (e.g., sheep) or faeces (e.g., cats); for Yersinia and Campylobacter, also faeces and environmental samples are admitted in the truth tables.

Echinococcus: Echinococcosis is one of the diseases listed in the Annex I part A, therefore it shall be monitored by the official competent authorities. In Italy, surveillance is performed during official meat inspection as part of Regulation (EC) No 854/2004 of the European Parliament and of the Council of 29th April 2004 laying down specific rules for the organisation of official controls on products of animal origin intended for human consumption [17]. Some regional programmes are in place on the national territory and official sampling activities take place at slaughterhouse as part of the post-mortem examination for all farmed animals. Therefore for echinococcosis the truth table admits the context "monitoring", sampler "official sampling", sampling strategy "census" and sampling stage "at slaughterhouse", according to Regulation (EC) No. 854/2004. The sample type admitted is "animal sample-organ/tissue". The sample unit is always "animal" since the unit tested in the framework of the monitoring programme is always the animal.

Brucella: The truth table for brucellosis does not admit official sampling activities carried out on cattle, sheep and goats and farmed water buffaloes, since these data are provided by the Italian information system on Community co-financed eradication programmes (SIR). Clinical investigations are admitted for other susceptible species, with sampling strategy "suspect sampling": for instance, pets may be subjected to clinical investigations carried out in case of suspect of disease, especially for dogs living on a farm. The sampling context "survey" is admitted for animal species susceptible to Brucella, with sampler "objective sampling". For sample type, typically blood is tested by serology; other animal samples could include abortion material. Sampling stage "natural habitat" is admitted for wild animals and "at farm" for farmed or zoo animals.

Trichinella: According to Regulation (EC) n. 2075/2005, the competent authority should implement a monitoring programme covering domestic swine, horses and other susceptible animal species coming from holdings or categories of holdings recognised as free from Trichinella or from regions where the risk of Trichinella in domestic swine is recognised as negligible, in order to verify that the animals are effectively free from Trichinella. To that end, meat samples shall be collected and examined for presence of Trichinella parasites. The truth table developed for trichinosis in animals admits only "animal" as sampling unit, "monitoring" as sampling context, "official sampling" as sampler and "census sampling" as sampling strategy. Since trichinosis is asymptomatic in most of the animal species, the context "clinical investigations" is not admitted.

\section{Discussion}

Data reported by the Member States and other reporting countries annually are stored in the EFSA's Zoonoses databases and used for the production of the annual European Union Summary Report (EUSR), other scientific or technical reports $[18,19]$, and for supporting risk analysis carried out by EFSA's scientific panels [20,21]. Since changes in the historical datasets of Member States occurred over the years and the standardisation of the data reporting improved during the past years, needs to update and complement the national historical datasets in the EFSA's Zoonoses databases were required. This was to guarantee the correctness of the data and the subsequent accuracy of the analyses based on the national data. The work done during the Grant project permitted to trace, wherever possible, the missing right information and to improve, overall, the quality of data reported to the EFSA from 2008 to 2011. The need to retrieve historical data led to improve the rules of the national data collection foreseen by the truth tables and to define new logics and algorithms that may be used and improved for each reporting.

In conclusion, the work done during the project permitted to trace, wherever possible, the missing right information and to improve, overall, the quality of data reported to the EFSA from 2008 to 2011. The Italian team developed an expert, flexible system, using business rules (i.e., truth tables) able to support the annual collection both at aggregate level and sample based level. From the Grant project until the last reporting season ( 2015 zoonoses data collection) the implementation of the truth tables in SINZoo allowed checking the correctness of the data reported, thus avoiding rough mistakes during the system's feeding. To date, the truth tables contain all the valid combination of context, stage, sampling unit allowed for a given zoonosis in a given area (animal, food, and feed) and for a category of species. During zoonoses data entry, the truth table checks that, for each zoonosis, the combination of the area of interest, each possible sampling context, stage and sampling unit has been entered correctly, thus avoiding inconsistent data. Moreover, the truth tables are under revision annually by the national Panel of expert: the revision takes into account any possible change in the national or EU legislation and also the addition of new combinations for which the experts decide the cogency.

In the absence of any structured information system, data collection would be prone to delays, errors and omissions. In fact, the same information could be requested several times and in different formats, causing confusion for the lack of a unique data collection.

This Grant project highlighted the importance of data quality during the collection and feeding of any information system. Data quality is the pillar for any epidemiological analysis and to perform risk analysis: logical rules of truth tables can be implemented in other information systems involved in data collection in the field of animal health and food safety, increasing both the consistence and the coherence of the data reported.

Ensure the quality of the data collected is the basis for any subsequent processing. For this reason, the efforts go towards the integration of the various information systems, avoiding duplication of the same data in different systems. The data is entered only in one spot, usually by those who created it and is made available to those who need it (interoperability). Moreover, the existing national information systems shifted their ability over time from operational applications (collecting data in course of normal business operation) to decision support systems having primarily the purpose to collect appropriate data of high quality and presenting the results of data analysis to stakeholders and decision makers.

\section{Acknowledgements}


Citation: Iannetti S, Cioci D, Falcone MG, Colangeli P (2017) The Zoonosis Data Collection in Italy: An Expert System for Data Quality Management and Improvement. J Vet Sci Technol 8: 431. doi: 10.4172/2157-7579.1000431

The authors would like to thank the European Food Safety Authority for funding the Grant project "Implementation and testing of electronic submission in XML, Excel and CSV formats of zoonoses, antimicrobial resistance and food-borne outbreak data and updating the historical dataset" under which this research was carried out.

\section{References}

1. European Parliament, Council of the European Union (2003) Directive 2003/99/ EC of the European Parliament and of the Council of 17 November 2003 on the monitoring of zoonoses and zoonotic agents, amending Council Decision 90/424/EEC and repealing Council Directive 92/117/EEC, pp: 31-40.

2. European Parliament, Council of the European Union (2002) Regulation (EC) No 178/2002 of the European Parliament and of the Council of 28 January 2002 laying down the general principles and requirements of food law, establishing the European Food Safety Authority and laying down procedures in matters of food safety, pp: 1-24.

3. EFSA (European Food Safety Authority) (2010) Standard sample description for food and feed. EFSA Journal 8: 1457.

4. Legislative Decree No. 191 of 4th April (2006) Implementation of Directive 2003/99/EC on the monitoring of zoonoses and zoonotic agents. Italian Official Gazette no. 119 of 24 May 2006.

5. Colangeli P, lannetti S, Ruocco L, Forlizzi L, Cioci D, et al. (2012) The Italian Information System on Zoonoses Data Collection. Zoonoses Public Health 60: 182-188.

6. European Food Safety Authority (EFSA) (2014) Manual for reporting on zoonoses and zoonotic agents, within the framework of Directive 2003/99/EC and on some other pathogenic microbiological agents for information deriving from the year 2015. 573: 107

7. Colangeli P, lannetti S, Cerella A, Ippoliti C, Di Lorenzo A, et al. (2011) The National information system for the notification of animal diseases in Italy. Vet Ital 47: 303-312.

8. Iannetti S, Malizia E, Sorgente A, Ricci A, Cibin V, et al. (2015) The Integrated Management System for Salmonella Control Plan in Italy. J Health Med Informat 6: 199.

9. Napoli C, lannetti S, Rizzo C, Bella A, DiSabatino D, et al. (2014) Vector borne infections in Italy: results of the integrated surveillance system for West Nile Disease in 2013. BioMed Res International. 2015: Article ID 643439.

10. Commission Decision No. 2007/851/EC of 10 December 2007 amending Decisions 2006/687/EC, 2006/875/EC and 2006/876/EC as regards the reallocation of the Community's financial contribution to certain Member States for their programmes for the eradication and monitoring of animal diseases and for checks aimed at the prevention of zoonoses for 2007 (notified under document number C (2007) 5985).
11. Commission implementing Decision No. 2011/807/UE of 30 November 2012 approving annual and multiannual programmes and the financial contribution from the Union for the eradication, control and monitoring of certain animal diseases and zoonoses presented by the Member States for 2013 and following years.

12. Commission Regulation (EC) No. $1003 / 2005$ implementing Regulation (EC) No. 2160/2003 of the European Parliament and of the Council as regards a Union target for the reduction of the prevalence of certain Salmonella serotypes in breeding flocks of Gallus gallus and amending Regulation (EC) No. 2160/2003.

13. Commission Regulation (EU) No. 1190/2012 of 12 December 2012 concerning a Union target for the reduction of Salmonella Enteritidis and Salmonella Typhimurium in flocks of turkeys, as provided for in Regulation (EC) No 2160/2003 of the European Parliament and of the Council Text with EEA relevance.

14. Commission Regulation (EC) No. 646/2007 of 12 June 2007 implementing Regulation (EC) No 2160/2003 of the European Parliament and of the Council as regards a Community target for the reduction of the prevalence of Salmonella Enteritidis and Salmonella Typhimurium in broilers and repealing Regulation (EC) No 1091/2005

15. Commission Decision No. 2007/848/EC of 11 December 2007 approving certain national programmes for the control of Salmonella in flocks of laying hens of Gallus gallus.

16. Commission Regulation (EC) No. 2075/2005 of 5 December 2005 laying down specific rules on official controls for Trichinella in meat.

17. Regulation (EC) of the European Parliament and of the Council No. 854/2004 (EC, 2004) of 29th April 2004 laying down specific rules for the organisation of official controls on products of animal origin intended for human consumption.

18. EFSA (European Food Safety Authority) and ECDC (European Centre for Disease Prevention and Control) (2016) The European Union summary report on trends and sources of zoonoses, zoonotic agents and food-borne outbreaks in 2015. EFSA Journal. 14: 04634.

19. European Food Safety Authority (EFSA), Boelaert F, Hugas M, Ortiz Pelaez A, Rizzi V, Stella P et al. (2016) The European Union summary report on data of the surveillance of ruminants for the presence of transmissible spongiform encep halopathies (TSEs) in 2015. EFSA Journal. 14: 4643.

20. Opinion of the Scientific Panel on Biological Hazards on "Risk assessment of a revised inspection of slaughter animals in areas with low prevalence of Trichinella". The EFSA Journal 200: 1-411.

21. Scientific Opinion of the Panel on Biological Hazards on a request from EFSA on Surveillance and monitoring of Toxoplasma in humans, foods and animals (2007) The EFSA Journal 583: 1-64. 